\title{
ON MONOCOMPOSITION ALGEBRAS
}

\author{
SEBASTIAN WALCHER
}

(Communicated by Lance W. Small)

\begin{abstract}
Monocomposition algebras satisfy a weaker version of composition with a certain quadratic form. The main result of this paper is that the construction of unital nondegenerate monocomposition algebras requires a family of skew-symmetric linear maps with certain additional conditions. Some classes of examples are discussed.
\end{abstract}

Introducing a generalization of composition algebras, Gainov [1] called an algebra $A$ over a field $K$ together with a nonzero quadratic form $N$ on $A$ a monocomposition algebra if the identity

$$
N\left(x^{2}\right)=N(x)^{2}
$$

holds for all $x \in A$. We will always assume that the characteristic of $K$ is different from 2 and 3 . As the defining identity involves only the commutative part $A^{+}$of $A$, and $A^{-}$is arbitrary, it seems appropriate to require commutativity of $A$, as we will do from now on. Furthermore we will only consider finite-dimensional monocomposition algebras. A monocomposition algebra is called (non-)degenerate if the quadratic form $N$ has this property. Borrowing the familiar terminology from composition algebras we call $N$ a norm of $A$.

Examples of monocomposition algebras abound. For instance, every quadratic unital Jordan algebra, satisfying an identity $x^{2}+t(x) x+n(x) 1=0$, is monocomposition with norm $-n$. Many more examples were found by Gainov $[1,2,3,4,5]$. (It should be noted that only those articles by Gainov with direct relevance to the present work are listed here.) Another class of (nonunital) monocomposition algebras was recently investigated by Meyberg and Osborn [6], who exhibited an interesting connection between these and central simple Jordan algebras of degree 3 . While this class of examples indicates that unitality is not a necessary ingredient of "interesting" monocomposition algebras, we will only deal with the unital case here.

Even for unital monocomposition algebras, anything even resembling a classification seems beyond reach, and worse, there is no way known to construct them all (while for composition algebras the necessary ingredients are familiar). The purpose of this article is to show that there is at least a natural first step towards their construction. This is proved in Theorem 3, and the algebras involved in this first step are then investigated in some detail. We will also discuss

Received by the editors November 8, 1993.

1991 Mathematics Subject Classification. Primary 17A42, 17A75, 17 A45.

(C) 1995 American Mathematical Society 
two classes of examples which, from the viewpoint of Theorem 3, seem to give rise to the "simplest" nontrivial unital monocomposition algebras.

The defining identity (M1) has degree four while $K$ has at least five elements. This fact allows us to linearize (M1) to obtain the (equivalent) identities

$$
\begin{gathered}
N\left(x^{2}, x y\right)=N(x) N(x, y) ; \\
N\left(x^{2}, y z\right)+2 N(x y, x z)=N(x) N(y, z)+2 N(x, y) N(x, z) .
\end{gathered}
$$

(Here and in the following a quadratic form and its associated symmetric bilinear form will not be distinguished typographically.)

Now let $A$ be a unital monocomposition algebra. Then $N(1)=1$ follows easily from (M1) and its linearizations (Gainov [2]), and we have an orthogonal direct sum $A=K \cdot 1+B$, with $B=(K \cdot 1)^{\perp}$. Obviously there is a quadratic form $\varphi$ on $B$ and a commutative multiplication "o" on $B$ such that for $x=$ $\xi \cdot 1+x_{0}$ and $y=\eta \cdot 1+y_{0} \in A$ (with $\xi, \eta \in K$ and $x_{0}, y_{0} \in B$ ) we have

$$
\left(\xi \cdot 1+x_{0}\right)\left(\eta \cdot 1+y_{0}\right)=\left(\xi \eta+\varphi\left(x_{0}, y_{0}\right)\right) \cdot 1+\left(\xi y_{0}+\eta x_{0}+x_{0} \circ y_{0}\right) .
$$

This natural approach (similar to the consideration of trace zero elements in composition algebras) is due to Gainov [1], as is the following result.

Proposition 1. (a) Let $A$ be a unital monocomposition algebra with norm $N$ and $(B, \circ, \varphi)$ as above. Then $N\left(\xi \cdot 1+x_{0}\right)=\xi^{2}-\varphi\left(x_{0}\right)$ for all $x=\xi \cdot 1+x_{0} \in A$, and the identities $\varphi\left(x_{0}, x_{0} \circ x_{0}\right)=\varphi\left(x_{0} \circ x_{0}\right)=0$ hold for all $x_{0} \in B$.

(b) Conversely, let $(B, \circ)$ be a commutative algebra and $\varphi$ a quadratic form on $B$ such that $\varphi\left(x_{0}, x_{0} \circ x_{0}\right)=\varphi\left(x_{0} \circ x_{0}\right)=0$ for all $x_{0} \in B$. On $A=K \cdot 1+B$ define a commutative product by $\left(\xi \cdot 1+x_{0}\right)^{2}:=\left(\xi^{2}+\varphi\left(x_{0}\right)\right) \cdot 1+\left(2 \xi x_{0}+x_{0} \circ x_{0}\right)$ and a quadratic form $N$ by $N\left(\xi \cdot 1+x_{0}\right):=\xi^{2}-\varphi\left(x_{0}\right)$. Then $A$ is a unital monocomposition algebra with norm $N$.

This shows that studying unital monocomposition algebras is the same as studying commutative algebras $(B, \circ)$ equipped with a quadratic form $\varphi$ such that the following identities hold:

$$
\begin{gathered}
\varphi(x, x \circ x)=0 \\
\varphi(x \circ x)=0 .
\end{gathered}
$$

(Gainov [2] calls such algebras quasi-monocomposition algebras.)

Given arbitrary $\varphi$, the easiest choice for the product on $B$ is the trivial product $(B \circ B=\{0\})$, which gives rise to the quadratic unital Jordan algebras already mentioned. As another extreme case, given any commutative algebra $(B, \circ)$, take $\varphi=0$. Then $N$ is just the square of a homomorphism from $A$ to $K$. To avoid such pathological cases we will from now on require $\varphi$ to be nondegenerate (and therefore restrict our interest to nondegenerate monocomposition algebras).

Our first result shows that there is a rather precise description of nontrivial algebras $(B, \circ)$ satisfying $(\mathrm{S} 1)$ and $(\mathrm{S} 2)$ for which the dimension of $B \circ B$ is as small as possible.

Proposition 2. Let $K$ be infinite, and let $(B, \circ, \varphi)$ satisfy (S1) and (S2). If $B \circ B \neq\{0\}$, then $\operatorname{dim}(B \circ B) \geq 2$. Furthermore, if $\operatorname{dim}(B \circ B)=2$, then there 
exist a linear form $\mu$ on $B$ and a linear $f: B \rightarrow B$ which is skew-symmetric with respect to $\varphi$, has rank 2 and satisfies $f^{2}=0$ such that

$$
x \circ x=\mu(x) f(x) \text { for all } x \in B \text {. }
$$

Proof. Because $K$ is infinite, the algebra of polynomial functions from $B$ to $K$ has no zero divisors and is a unique factorization domain.

Assume that $\operatorname{dim}(B \circ B)=1$. Then there exist $c \in B$ and a quadratic form $\rho$ on $B$, both nonzero, such that $x \circ x=\rho(x) c$ for all $x$. From (S1) we find $0=\varphi(x, x \circ x)=\rho(x) \varphi(x, c)$ for all $x$, hence $\varphi(x, c)=0$ for all $x$, a contradiction to the nondegeneracy of $\varphi$. This shows $\operatorname{dim}(B \circ B) \geq 2$.

Now let $\operatorname{dim}(B \circ B)=2$. Then there exists linearly independent $c_{1}, c_{2}$ in $B$ and linearly independent quadratic forms $\rho_{1}, \rho_{2}$ on $B$ such that

$$
x \circ x=\rho_{1}(x) c_{1}+\rho_{2}(x) c_{2} \text { for all } x \in B .
$$

From $(\mathrm{S} 1)$ we get $\rho_{1}(x) \varphi\left(x, c_{1}\right)+\rho_{2}(x) \varphi\left(x, c_{2}\right)=0$. The linear forms $\varphi\left(x, c_{1}\right)$ and $\varphi\left(x, c_{2}\right)$ are linearly independent (due to the linear independence of $c_{1}$ and $c_{2}$ and the nondegeneracy of $\varphi$ ), hence relatively prime. Therefore the relation above shows that $\varphi\left(x, c_{1}\right)$ divides $\rho_{2}(x), \varphi\left(x, c_{2}\right)$ divides $\rho_{1}(x)$, and furthermore $\rho_{1}(x)=\mu(x) \varphi\left(x, c_{2}\right), \rho_{2}(x)=-\mu(x) \varphi\left(x, c_{1}\right)$ for some linear form $\mu$. Define the linear map $f$ by $f(x)=\varphi\left(x, c_{2}\right) c_{1}-\varphi\left(x, c_{1}\right) c_{2}$. Clearly $f$ is skew-symmetric with respect to $\varphi$, has rank 2 , and $x \circ x=\mu(x) f(x)$ holds.

Finally, (S2) implies

$$
0=\varphi(x \circ x)=\mu(x)^{2} \varphi(f(x))=-\mu(x)^{2} \varphi\left(x, f^{2}(x)\right),
$$

therefore $\varphi\left(x, f^{2}(x)\right)=0$ for all $x$ and $f^{2}=0$ follows, as $f^{2}$ is symmetric with respect to $\varphi$.

Even if the condition on the rank of $f$ is discarded, every algebra constructed according to the above proposition satisfies (S1) and (S2). The algebras in this larger class are distinguished by the property of having a trivial subalgebra of codimension one (viz. the kernel of $\mu$ ), and from this point of view they (resp. the monocomposition algebras corresponding to them) are discussed by Gainov $[1$, p. 10]. An obvious generalization of this construction is the following:

Given $(B, \varphi)$ let $\left(f_{1}, \ldots, f_{r}\right)$ be a family of skew-symmetric linear maps satisfying $f_{i} f_{j}+f_{j} f_{i}=0$ for all $i, j \in\{1, \ldots, r\}$, and let $\mu_{1}, \ldots, \mu_{r}$ be linear forms. Define a commutative multiplication via $x \circ x:=\sum_{i=1}^{r} \mu_{i}(x) f_{i}(x)$. Then $(B, \circ, \varphi)$ satisfies $(\mathrm{S} 1)$ and $(\mathrm{S} 2)$. This class is also discussed by Gainov [1, Theorem 7], although presented in a somewhat different manner, and provides one motivation to search for "families of anticommuting (skew-symmetric) matrices"; cf. Gainov [4, 5].

Actually, these algebras can be seen from a different perspective. Define a noncommutative product " $*$ " on $B$ via

$$
x * y:=\sum_{i=1}^{r} \mu_{i}(x) f_{i}(y) .
$$

Then clearly $(B, \circ)=(B, *)^{+}$, and $(B, *, \varphi)$ satisfies the stronger identities

$$
\varphi(x * y, z)=-\varphi(y, x * z)
$$


and

$$
\varphi(x * y, x * z)=0 .
$$

This raises the question whether considering noncommutative products on $B$ with stronger properties can provide more insight. (Of course, the commutative part should still be $(B, \circ)$ !) More precisely, let us ask: Given $(B, *, \varphi)$ satisfying (S1), is there a product " $*$ " on $B$ such that $(B, *)^{+}=(B, 0)$ and that $(B, *, \varphi)$ satisfies (S1\#)? Obviously, if there is such a product, then it is not, in general, uniquely determined, and there is also the problem of singling out a unique product with nice properties, should any exist.

The following theorem gives an answer to the existence and uniqueness problems. Note that the assumption on the characteristic of $K$ is essential here.

Theorem 3. Suppose that $(B, \circ, \varphi)$ satisfies $(\mathrm{S} 1)$. Then there is a multiplication “*” on $B$ which is uniquely determined by $(B, *)^{+}=(B, \circ)$ and

$$
\varphi(x * y, z)-\varphi(y * x, z)=\varphi(x, z * y)
$$

for all $x, y, z \in B$. In particular, $(B, *, \varphi)$ satisfies $(\mathrm{S} 1 \#)$. Furthermore

$$
2 \varphi(x \circ y, z)+4 \varphi(x, y \circ z)=3 \varphi(x, z * y)
$$

holds for all $x, y, z \in B$.

Proof. (i) First assume that $(B, *, \varphi)$ satisfies $(\mathrm{A} 1)$ and $(B, *)^{+}=(B, 0)$. Then (S1\#) follows from (A1) for $x=y$. Using (A1) and (S1\#) and $2 x \circ y=$ $x * y+y * x$, we get

$$
\begin{aligned}
\varphi(x, z * y) & =\varphi(x * y+y * x, z)-2 \varphi(y * x, z) \\
& =2 \varphi(x \circ y, z)+2 \varphi(x, y * z) \\
& =2 \varphi(x \circ y, z)+2 \varphi(x, y * z+z * y)-2 \varphi(x, z * y) \\
& =2 \varphi(x \circ y, z)+4 \varphi(x, y \circ z)-2 \varphi(x, z * y),
\end{aligned}
$$

and this is (A2). Uniqueness follows from (A2) and the nondegeneracy of $\varphi$.

(ii) If $(B, \circ, \varphi)$ is given and satisfies (S1), use (A2) to define the product "*" on $B$. Then a simple calculation yields

$$
3 \varphi(z * y-y * z, x)=2 \varphi(x \circ y, z)-2 \varphi(x \circ z, y) \text {. }
$$

Add the linearization of (S1),

$$
0=2 \varphi(x \circ y, z)+2 \varphi(x \circ z, y)+2 \varphi(y \circ z, x),
$$

to obtain

$$
3 \varphi(z * y-y * z, x)=2 \varphi(y \circ z, x)+4 \varphi(z, x \circ y)=3 \varphi(z, x * y)
$$

by (A2), and hence $(B, *, \varphi)$ satisfies (A1).

It remains to show that $(B ; *)^{+}=(B, \circ)$. Let $y=z$ in (A2) to see $3 \varphi(x, z * z)=4 \varphi(x, z \circ z)+2 \varphi(x \circ z, z)$. Using $2 \varphi(x \circ z, z)+\varphi(x, z \circ z)=0$ (linearization of $(\mathrm{S} 1))$, we have $3 \varphi(x, z * z)=3 \varphi(x, z \circ z)$, and $z * z=z \circ z$ follows from the nondegeneracy of $\varphi$.

Remarks. (a) A weaker version of this result (with a different proof) is given in [8, Theorem 5.8], where the connection with nondegenerate quadratic first integrals of ordinary differential equations is exhibited. 
(b) We have only used that $(B, \circ, \varphi)$ satisfies $(\mathrm{S} 1)$. It is easy to verify that the unital algebra $A$ and the quadratic form $N$ constructed from $(B, \circ, \varphi)$ as in Proposition 1(b) satisfy the identity $N\left(x, x^{2}\right)=N(x, 1) N(x)$ (an immediate consequence of (M2)), and that there is, once more, a 1-1-correspondence between algebras $(A, N)$ satisfying this identity and algebras $(B, \circ, \varphi)$ satisfying (S1).

(c) A consequence of the theorem is that for given $(B, \varphi)$ every algebra satisfying (S1) can be obtained in the following way: There are linear endomorphisms $f_{1}, \ldots, f_{r}$ of $B$ which are skew-symmetric with respect to $\varphi$ and linear forms $\mu_{1}, \ldots, \mu_{r}$ such that $x \circ x=\sum_{i=1}^{r} \mu_{i}(x) f_{i}(x)$. (So here we have a natural first step in the construction of unital monocomposition algebras.) This is a consequence of the fact that there is a product "•" on $B$ such that $(B, \bullet)^{+}=(B, \circ)$, and every left multiplication in $(B, \bullet)$ is skew-symmetric due to $(\mathrm{S} 1 \#)$. Of course, if one constructs $(B, \circ)$ from such a product, then "*" and "•" will in general be different. A simple calculation involving (A2) shows that the distinguished product is given by

$$
3 x * y=\sum_{i=1}^{r}\left(\varphi\left(f_{i}(y), x\right) c_{i}+\mu_{i}(y) f_{i}(x)+2 \mu_{i}(x) f_{i}(y)\right),
$$

where $c_{i}$ is defined by $\mu_{i}(x)=\varphi\left(x, c_{i}\right)$.

(d) Let $[x, y]:=x * y-y * x$ for $x, y \in B$. Then (A1) can be written as $\varphi([x, y], z)=\varphi(x, z * y)$, and in particular $(+) \varphi([x, z], z)=\varphi(x, z \circ z)$. This shows that $(B, \circ)$ can be obtained from the anticommutative part of $(B, *)$. This anticommutative algebra is uniquely determined by $(+)$ and $\varphi([x, y], z)+\varphi([z, x], y)+\varphi([y, z], x)=0$. Of course, every anticommutative algebra, via $(+)$, yields an algebra $(B, \circ)$ satisfying $(\mathrm{S} 1)$.

Before turning to $(\mathbf{S} 2)$ let us investigate in greater detail algebras $(B, \circ, \varphi)$ satisfying $(\mathrm{S} 1)$ and the related algebras $(B, *, \varphi)$ satisfying (A1). (In effect this amounts to an investigation of algebras $(A, N)$ satisfying $N\left(x, x^{2}\right)=$ $N(x, 1) N(x)$.

The (perhaps strange-looking) identity (A1) has some nice consequences. The following is an illustration, showing that (A1) is at least as powerful as an associative, nondegenerate bilinear form for the algebra.

Proposition 4. Let $(B, *, \varphi)$ satisfy (A1).

(a) If $J$ is an ideal of $B$, then $J^{\perp}$ is also an ideal, and $J * J^{\perp}=J^{\perp} * J=\{0\}$.

(b) $B$ is a direct sum of simple ideals if and only if $I * I \neq\{0\}$ for every nonzero ideal $I$ of $B$.

Proof. We start by establishing a few facts.

(i) If $J$ is a left ideal of $B$, then so is $J^{\perp}$. This is an immediate consequence of (S1\#).

(ii) If $J$ is a right ideal of $B$, then $J * J^{\perp}=\{0\}$. To see this, let $x \in J, y \in$ $J^{\perp}$ and use $\varphi(x * y, z)=-\varphi(y, x * z)$.

(iii) If $J$ is a right ideal of $B$, then $x * y-y * x \in J^{\perp}$ for all $y \in B$ and $x \in J^{\perp}$. To verify this, let $z \in J$ and $x \in J^{\perp}$ in (A1).

Now we can prove (a). Let $J$ be an ideal of $B$. Then $J^{\perp}$ is a left ideal by (i), and furthermore (due to (iii)) $x * y-y * x \in J^{\perp}$ for all $y \in B$ and $x \in J^{\perp}$, hence $x * y \in J^{\perp}$ for all $y \in B, x \in J^{\perp}$ and $J^{\perp}$ is a right ideal. Finally, (ii) implies $J * J^{\perp}=J^{\perp} * J=\{0\}$. Using (a)-a property well-known for algebras 
admitting a nondegenerate associative bilinear form-the proof of $(b)$ is exactly the same as in this familiar case (cf. Schafer [7, Theorem 2.6]).

Actually (A1) has even stronger consequences, like the following: If $J$ is a left ideal of $B$ and $\left.\varphi\right|_{J}$ is nondegenerate, then $J$ is already an ideal. To see this, let $x \in J, y \in J^{\perp}$ in (A1) to conclude $x * y=y * x \in J \cap J^{\perp}=\{0\}$, hence $J * J^{\perp}=J^{\perp} * J=\{0\}$, which is sufficient.

Of course, the structure of $(B, \circ)$ is of primary interest, but the following shows that it is closely tied to the structure of $(B, *)$.

Proposition 5. Let $(B, *, \varphi)$ satisfy $(\mathrm{A} 1)$, and $(B, \circ)=(B, *)^{+}$. Then the following are true:

(a) If $I$ is an ideal of $(B, \circ)$, then $I^{\perp}$ is a subalgebra of $(B, *)$ and $I * I^{\perp}+I^{\perp} * I \subset I$. If $\left.\varphi\right|_{I}$ is nondegenerate, then $I$ is an ideal of $(B, *)$ iff $I * I^{\perp}=I^{\perp} * I=\{0\}$ iff $I \circ I^{\perp}=\{0\}$.

(b) Let $I$ and $J$ be ideals of $(B, \circ)$ such that $B=I \oplus J$, and further suppose $I \circ I=I$. Then $J=I^{\perp}$, and $I$ and $J$ are ideals of $(B, *)$.

(c) If $(B, \circ)$ is a direct sum of simple ideals, then this is an orthogonal direct sum and $(B, *)$ is the direct sum of these (simple) ideals.

(d) Let $I$ be a subspace of $B$. Then $B \circ I=\{0\}$ iff $B * I=I * B=\{0\}$.

Proof. (a) Let $y \in I$ and $z, x \in I^{\perp}$. Then (A2) yields $0=3 \varphi(x, z * y)=$ $-3 \varphi(z * z, y)$, showing $I^{\perp} * I \subset I$ and $I^{\perp} * I^{\perp} \subset I^{\perp}$. Now (A1) shows $0=\varphi(x * y-y * x, z)$, hence $x * y \in I$ implies $y * x \in I$, and $I * I^{\perp} \subset I$.

If $I$ is an ideal of $(B, *)$, then $I * I^{\perp}=I^{\perp} * I=I \circ I^{\perp}=\{0\}$ is immediate from Proposition 4. Conversely, suppose $I \circ I^{\perp}=\{0\}$. Since $B$ is the direct sum of the vector spaces $I$ and $I^{\perp}$ and $I * I^{\perp}+I^{\perp} * I \subset I$ is already known, it remains to show that $I$ is a subalgebra of $(B, *)$. But for $x, z \in I$ and $y \in I^{\perp}$ we get

$$
-3 \varphi(z * x, y)=3 \varphi(x, z * y)=2 \varphi(x \circ y, z)+4 \varphi(x, y \circ z)=0
$$

from (A2), since $x \circ y=y \circ z=0$, and $z * x \in I$ follows.

(b) Use the linearization of (S1)

$$
\varphi(x \circ y, z)+\varphi(z \circ x, y)+\varphi(y \circ z, x)=0
$$

with $x, z \in I, y \in J$ (hence $x \circ y=y \circ z=0$ ) to obtain $\varphi(z \circ x, y)=0$. Hence $0=\varphi(I \circ I, J)=\varphi(I, J)$ and $J \subset I^{\perp}$; finally $J=I^{\perp}$ by a dimension argument.

(c) This is an obvious consequence of (b).

(d) Suppose $B \circ I=\{0\}$ and use (A2) with $y \in I$ to find $\varphi(x, z * y)=0$ for all $x, z \in B$, or $B * I=\{0\}$. Now $I * B=\{0\}$ follows from $B \circ I=\{0\}$.

It is not generally true that every ideal of $(B, \circ)$ is also an ideal of $(B, *)$; counterexamples are easily found in the class of algebras discussed in Proposition 2. It is not clear whether (semi-)simplicity of $(B, *)$ implies (semi-) simplicity of $(B, \circ)$, although this seems unlikely. But (d) shows, for instance, that $(B, *)$ cannot be semisimple when $(B, \circ)$ is nilpotent.

So far we have only exploited (S1); let us turn to (S2) now. It is not true that for every $(B, \circ, \varphi)$ satisfying $(\mathrm{S} 1)$ and $(\mathrm{S} 2)$ there is an algebra $(B, \bullet)$ that satisfies $(\mathbf{S} 1 \#),(\mathbf{S} 2 \#)$ and $(B, \bullet)^{+}=(B, \circ)$. In fact, every algebra with this latter property satisfies $(x \circ x) \circ(x \circ x)=0$, as is readily verified, while there 
are algebras satisfying (S1) and (S2) but not this identity (and are not even "monosolvable", cf. Gainov [3]).

Having thus ensured that it is hopeless to search for algebras $(B, \bullet)$ satisfying $(\mathrm{S} 1 \#)$ and $(\mathrm{S} 2 \#)$ for any given $(B, \circ)$, we return to algebras satisfying $(\mathrm{A} 1)$ and see what condition $(\mathrm{S} 2)$ for $(B, \circ, \varphi)$ means.

Proposition 6. Let $(B, *, \varphi)$ satisfy $(\mathrm{A} 1)$, and let $(B, \circ)=(B, *)^{+}$. Then $(B, \circ, \varphi)$ satisfies $(\mathrm{S} 2)$ if and only if

$$
2 x *(x * x)=(x * x) * x
$$

for all $x \in B$.

Proof. Suppose that (S2) holds; then

$$
\varphi(x * y, x * x)+\varphi(y * x, x * x)=0
$$

follows for all $x \in B$, while (A1) implies

$$
\varphi(x * y, x * x)-\varphi(y * x, x * x)=\varphi(x,(x * x) * y) .
$$

Adding these and using (S1\#) yields

$$
-2 \varphi(y, x *(x * x))=-\varphi((x * x) * x, y),
$$

hence the asserted identity. Conversely, this identity and (S1\#) imply

$$
-2 \varphi(x * x, x * x)=2 \varphi(x, x *(x * x))=\varphi(x,(x * x) * x)=0 .
$$

Here we have all the trouble with the construction of monocomposition algebras concentrated in one (strange) identity. We have seen the connection to algebras satisfying (A1) and these, in general, are easy to construct (recall remark (c) following Theorem 3) and have some nice structural properties. But the identity forced onto these algebras by (S2) singles out a rather pathological subclass.

Let us draw one consequence from this identity.

Corollary 7. Let $(B, \circ, \varphi)$ satisfy $(\mathrm{S} 1)$ and $(\mathrm{S} 2)$. Then $B$ contains no idempotent.

Proof. The idempotents of $(B, \circ)$ and $(B, *)$ are the same. Now $c * c=c$ implies

$$
2 c=2 c *(c * c)=(c * c) * c=c \text { and } c=0 .
$$

This result can also be obtained more directly from various linearizations of (S1) and (S2).

The results presented above may serve as starting points for the further investigation of monocomposition algebras. Here, just to illustrate how they can be applied, we will be content to construct one more class of examples.

By Theorem 3 we know that for every algebra $(B, \circ)$ satisfying (S1) there is a product "॰" on $B$ such that $(B, \bullet)^{+}=(B, \circ)$ and every left multiplication $L(x)$ in $(B, \bullet)$ is skew-symmetric with respect to $\varphi$. The algebras discussed in and immediately following Proposition 2 are then distinguished by the existence of a $(B, \bullet)$ so that the subspace $\{L(x): x \in B\}$ has dimension one. From this point of view it seems natural to consider the "next easiest" case with $\{L(x): x \in B\}$ two-dimensional for some $(B, \bullet)$. Again we require $K$ to be infinite. 
In other words, we now turn to algebras $(B, \circ)$ with

$$
x \circ x=\mu_{1}(x) f_{1}(x)+\mu_{2}(x) f_{2}(x) ;
$$

here $f_{1}$ and $f_{2}$ are linearly independent endomorphisms of $B$ which are skewsymmetric with respect to $\varphi$, and $\mu_{1}, \mu_{2}$ are linearly independent linear forms. This guarantees (S1), while (S2) is equivalent to the identity

$(*)$

$\mu_{1}(x)^{2} \varphi\left(x, f_{1}^{2}(x)\right)+\mu_{1}(x) \mu_{2}(x) \varphi\left(x,\left(f_{1} f_{2}+f_{2} f_{1}\right)(x)\right)+\mu_{2}(x)^{2} \varphi\left(x, f_{2}^{2}(x)\right)=0$.

If $f_{1}^{2}=f_{1} f_{2}+f_{2} f_{1}=f_{2}^{2}=0$, then we are back to $(\mathbf{S} 1 \#)$ and (S2\#) for $(\boldsymbol{B}, \bullet)$; we need not discuss this case any further here. Recall that $f_{1}^{2}, f_{1} f_{2}+f_{2} f_{1}$ and $f_{2}^{2}$ are symmetric with respect to $\varphi$, and let $\rho_{i}(x):=\varphi\left(x, f_{i}^{2}(x)\right)$ for $i=1,2$ and $\sigma(x):=\varphi\left(x,\left(f_{1} f_{2}+f_{2} f_{1}\right)(x)\right)$. Recall further the following elementary facts about quadratic forms: A quadratic form $\psi \neq 0$ (viewed as a polynomial) is irreducible whenever $\operatorname{rank}(\psi)>2$; in case $\operatorname{rank}(\psi)=2$ it is reducible iff it is hyperbolic. (In rank one we have $\psi=\alpha \lambda^{2}$ for some linear form $\lambda$ and some $\alpha \in K^{*}$.)

Now suppose $f_{2}^{2} \neq 0$, hence $\rho_{2} \neq 0$. Then $(*)$ implies that $\mu_{1}$ must divide $\rho_{2}$, and therefore $\operatorname{rank}\left(\rho_{2}\right)=\operatorname{rank}\left(f_{2}^{2}\right) \leq 2$. By the same token, $f_{1}^{2} \neq 0$ implies that $\mu_{2}$ divides $\rho_{1}$, and that $\operatorname{rank}\left(f_{1}^{2}\right)<2$. Therefore factorization arguments yield quite strong conditions on $f_{1}$ and $f_{2}$, and furthermore, for instance, $f_{2}^{2} \neq 0$ implies that there are (up to factors from $K^{*}$ ) at most two possible choices for $\mu_{1}$.

It would be inappropriate to discuss all the possible cases here; in the following we will focus on semisimple $f_{1}, f_{2}$ so that $(*)$ is satisfied for some $\mu_{1}, \mu_{2}$.

Semisimplicity implies $\operatorname{rank}\left(f_{1}^{2}\right)=\operatorname{rank}\left(f_{1}\right)$, and therefore $\operatorname{rank}\left(f_{1}\right)=2$ follows from skew-symmetry. Let $\left(c_{1}, c_{2}\right)$ be a basis of $U_{1}:=\operatorname{Im}\left(f_{1}\right)$. Then skev:'symmetry forces $f_{1}(x)=\alpha\left(\varphi\left(x, c_{2}\right) c_{1}-\varphi\left(x, c_{1}\right) c_{2}\right)$ for some $\alpha \in K^{*}$, and it is harmless to put $\alpha=1$. Now $B=U_{1}+\operatorname{Ker}\left(f_{1}\right)$ is a direct sum, showing $\varphi\left(v, c_{1}\right)=\varphi\left(v, c_{2}\right)=0$ for all $v \in \operatorname{Ker}\left(f_{1}\right)$, and the direct sum $U_{1}+\operatorname{Ker}\left(f_{1}\right)$ is orthogonal. Therefore $\left.\varphi\right|_{U_{1}}$ is nondegenerate. The equality $\rho_{1}(x)=-\varphi\left(f_{1}(x)\right)$ shows that $\left.\rho_{1}\right|_{U_{1}}$ is nondegenerate. Hyperbolicity of $\left.\rho_{1}\right|_{U_{1}}$ implies that there is a nonzero $w \in U_{1}$ such that $0=\rho_{1}(w)=-\varphi\left(f_{1}(w)\right)$, and therefore $\left.\varphi\right|_{U_{1}}$ is hyperbolic. (We have used the invertibility of $\left.f_{1}\right|_{U_{1}}$.) We may therefore assume that $\varphi\left(c_{1}\right)=\varphi\left(c_{2}\right)=0$ and $\varphi\left(c_{1}, c_{2}\right)=1$. This implies $f_{1}^{2}(x)=\varphi\left(x, c_{2}\right) c_{1}+\varphi\left(x, c_{1}\right) c_{2}$ and $\rho_{1}(x)=2 \varphi\left(x, c_{1}\right) \varphi\left(x, c_{2}\right)$. By the same arguments there is a basis $\left(d_{1}, d_{2}\right)$ of $U_{2}:=\operatorname{Im}\left(f_{2}\right)$ such that $f_{2}(x)=$ $\varphi\left(x, d_{2}\right) d_{1}-\varphi\left(x, d_{1}\right) d_{2}, \varphi\left(d_{1}\right)=\varphi\left(d_{2}\right)=0$ and $\varphi\left(d_{1}, d_{2}\right)=1$, implying $\rho_{2}(x)=2 \varphi\left(x, d_{1}\right) \varphi\left(x, d_{2}\right)$.

Finally, a brute force calculation shows

$$
\begin{aligned}
\sigma(x)= & 2 \varphi\left(x, d_{2}\right) \varphi\left(x, c_{1}\right) \varphi\left(d_{1}, c_{2}\right)-2 \varphi\left(x, d_{1}\right) \varphi\left(x, c_{1}\right) \varphi\left(c_{2}, d_{2}\right) \\
& -2 \varphi\left(x, d_{2}\right) \varphi\left(x, c_{2}\right) \varphi\left(c_{1}, d_{1}\right)+2 \varphi\left(x, d_{1}\right) \varphi\left(x, c_{2}\right) \varphi\left(c_{1}, d_{2}\right) .
\end{aligned}
$$

It follows from $(*)$ that there are essentially only two possible candidates for $\mu_{1}$, resp. $\mu_{2}$. We chose $\mu_{1}(x)=\varphi\left(x, d_{1}\right)$ and $\mu_{2}(x)=\alpha \varphi\left(x, c_{1}\right)$ for some $\alpha \in K^{*}$. (Symmetry considerations show that there is no loss of generality by the choice of $c_{1}$ and $d_{1}$; and the normalization of $\mu_{1}$ is harmless since 
changing the whole product by a nonzero scalar is harmless.) Then $(*)$ yields, after dividing by $2 \varphi\left(x, c_{1}\right) \varphi\left(x, d_{1}\right)$ :

$$
-\varphi\left(x, d_{1}\right) \varphi\left(x, c_{2}\right)-\alpha^{2} \varphi\left(x, c_{1}\right) \varphi\left(x, d_{2}\right)=\frac{1}{2} \alpha \sigma(x)
$$

We now make the additional assumption that the sum $U_{1}+U_{2}$ is direct (with basis $\left.\left(c_{1}, c_{2}, d_{1}, d_{2}\right)\right)$. Then the quadratic forms $\varphi\left(x, c_{i}\right) \varphi\left(x, d_{j}\right)$ are linearly independent, and comparing coefficients shows that $(*)$ holds if and only if

$$
\varphi\left(d_{1}, c_{2}\right)=-\alpha, \quad \varphi\left(c_{1}, d_{1}\right)=\varphi\left(c_{2}, d_{2}\right)=0 \text { and } \varphi\left(c_{1}, d_{2}\right)=-\alpha^{-1} .
$$

In other words, the matrix of $\left.\varphi\right|_{U_{1}+U_{2}}$ with respect to the above basis is given by

$$
\frac{1}{2}\left(\begin{array}{cccc}
0 & 1 & 0 & -\alpha^{-1} \\
1 & 0 & -\alpha & 0 \\
0 & -\alpha & 0 & 1 \\
-\alpha^{-1} & 0 & 1 & 0
\end{array}\right)
$$

This matrix has rank 2 for any choice of $\alpha$, therefore $\left.\varphi\right|_{U_{1}+U_{2}}$ is degenerate and nondegeneracy of $\varphi$ forces $\operatorname{dim}(B) \geq 6$.

On the other hand, on every vector space of dimension $\geq 6$ there are quadratic forms which have the desired properties. The product is then determined by

$x \circ x=\varphi\left(x, d_{1}\right) \varphi\left(x, c_{2}\right) c_{1}+\alpha \varphi\left(x, c_{1}\right) \varphi\left(x, d_{2}\right) d_{1}-\varphi\left(x, c_{1}\right) \varphi\left(x, d_{1}\right)\left(c_{2}+\alpha d_{2}\right)$.

It is easy to verify that $B \circ B$ is three-dimensional (spanned by $c_{1}, d_{1}$ and $\left.c_{2}+\alpha d_{2}\right)$ and that $B \circ B$ is a trivial ideal of $B$. A straightforward calculation yields the identity

$$
x \circ(x \circ(x \circ x))=\lambda(x) \cdot x \circ(x \circ x),
$$

with the linear form $\lambda$ defined by $\lambda(x)=\frac{1}{2} \varphi\left(x, d_{1}+\alpha c_{1}\right)$. This shows that $(B, \circ)$ is neither nil nor nilpotent.

While we have only discussed special types of this class of monocomposition algebras, it is quite clear how to tackle the remaining ones.

In this example we have only used part of the information contained in Theorem 3 , since $(*)$ can be handled well using divisibility arguments. In general, however, it seems useful to employ the distinguished product introduced in the theorem.

\section{REFERENCES}

1. A. T. Gainov, Monocomposition algebras, Siberian Math. J. 10 (1969), 1-21.

2. 1595-1598.

3. __ Subalgebras of nondegenerate commutative QM-algebras, Algebra Logika 15 (1976), no. 4, 371-383.

4. Anticommuting matrices and their application to monocomposition algebras, Siberian Math. J. 30 (1989), 877-882.

5. _ Maximal spaces of skew-symmetric and symmetric anticommutative matrices, Siberian Math. J. 32 (1991), no. 6, 924-933. 
6. K. Meyberg and J. M. Osborn, Pseudo-composition algebras, Math. Z. 214 (1993), 67-77.

7. R. D. Schafer, An introduction to nonassociative algebras, Academic Press, New York, 1966.

8. S. Walcher, Algebras and differential equations, Hadronic Press, Palm Harbor, 1991.

Mathematisches Institut, TU MÜNChen, 80290 MÜnChen, GeRMany

Current address: Department of Mathematics, New Mexico State University, Las Cruces, New Mexico 88003

E-mail address: swalcher@nmsu.edu 